Pacific Journal of Mathematics

THE SYMMETRY OF SESSILE AND PENDENT DROPS 


\title{
THE SYMMETRY OF SESSILE AND PENDENT DROPS
}

\author{
HenRy C. Wente
}

\begin{abstract}
Let $X$ denote a bounded, open, and connected subset of $R^{n+1}(n \geq 1)$ which we consider to represent the interior of a liquid drop (when $n=2$ ). The principal result of this paper will be to show that under suitable conditions $X$ is an axially symmetric drop in the sense that there is a vertical line (axis) such that any nonempty intersection of $X$ with a horizontal hyperplane is an open disk whose center lies on the axis. Condition 1: $X$ adheres to a horizontal hyperplane, $\Sigma$ (i.e., $X \cap \Sigma=\Phi$ but $\bar{X} \cap \Sigma \neq \Phi)$, with the mean curvature, $H$, of the liquid-air interface, $\Omega$, a differentiable function of the vertical coordinate and the angle of contact, $\alpha$, of $\Omega$ with $\Sigma$ a constant along $\partial \Omega$, $0 \leq \alpha \leq \pi$, (Theorem 1. 1). Condition 2: $X$ adheres to $\Sigma$ with the mean curvature a smooth function of height and the contact region of $\bar{X}$ with $\Sigma$ a disk (special case of Theorem 1.2).
\end{abstract}

1. Introduction. Let $\left(x_{1}, \cdots, x_{n}, u\right)$ be a Euclidean coordinate system for $R^{n+1}$. Theorem 1.1, which we now state, corresponds to the equilibrium state of a homogeneous pendent (or sessile) drop adhering to a horizontal hyperplane, $\Sigma$.

THEOREM 1.1. Let $X$ be a bounded, open, and connected subset of $R^{n+1}$ which is adhering to the hyperplane $\Sigma:\{u=0\}$. Suppose that the boundary of $X, \partial X=\Sigma_{X} \cup \Omega$ where $\Sigma_{X}=\Sigma \cap \bar{X}$ and $\Omega$, the liquid-air interface, is a hypersurface with boundary of class $C^{2}$ embedded in $R^{n+1}$ such that $\Gamma \equiv \partial \Omega=\Omega \cap \Sigma$. Suppose that the mean curvature, $H$, of $\Omega$ measured relative to the exterior normal is the restriction to $\Omega$ of a $C^{1}$-function on $R^{n+1}$ depending on the u-coordinate alone. Finally, suppose that the angle of contact, $\alpha$, of $\Omega$ with $\Sigma$ measured interior to $X$ is a constant along $\partial \Omega$ where $0 \leqq \alpha \leqq \pi$. Then there is a vertical line about which $X$ is axially symmetric such that any nonempty intersection of $X$ with a hor $i$ zontal hyperplane is an open disk with center on the axis.

The physical case of $X \subset R^{3}$ and mean curvature $H=-k u+c$ $(k>0)$ corresponds to a sessile drop when $X$ is above $\Sigma$, a pendent drop when $X$ is below $\Sigma$. The case $k=0$ of constant mean curvature is the situation of no gravity.

$J$. Serrin [8] treated the case where the liquid-air interface, $\Omega$, may be expressed nonparametrically, $u=u\left(x_{1}, \cdots, x_{n}\right)$ with $H$ a linear function of height. It turns out that the method of proof used there may be adapted to the present situation. The key tools are 
the E. Hopf maximum principle [5], the Hopf boundary point lemma [6], and Serrin's boundary point lemma at a corner [8]. We shall state these "lemmas" in $\S$ II. The method of proof is to take a vertical hyperplane, $T_{0}$, which initially lies outside of $\bar{X}$ and move it towards and into $X$ through the one parameter family of hyperplanes, $T$, parallel to $T_{0}$. As $T$ moves into $X$ one takes that part of $\Omega$ through which $T$ has passed and reflects it about $T$ forming the reflected surface, $\Omega^{\prime}(T)$. Initially $\Omega^{\prime}(T)$ lies inside $X$ and we look for a first time when this will fail. At this point one applies one of the touching lemmas to conclude that $\Omega^{\prime}(T)$ is identical to the unreflected portion of $\Omega$.

This device was first introduced by Alexandrov [1] who was able to show that the only embedded compact hypersurface of constant mean curvature is a sphere. The procedure was then refined by Serrin in [7] and [8].

I became interested in this problem through the work of $P$. Concus and R. Finn [2] who made a study of axially symmetric pendent drops. Their work induced the author to investigate the stability properties of such drops [9]. A detailed study of the axially symmetric sessile drop has recently been done by Finn [3]. I should also like to mention the paper of E. Gonzalez [4], in which it is proven that for any prescribed volume and any angle of contact $\alpha, 0<\alpha \leqq \pi$, there exists a sessile drop of minimum energy. By a symmetrization argument, such a drop must be axially symmetric. I am indebted to S.T. Yau who brought up the problem considered in this paper and suggested that the method of Alexandrov might work.

The question of symmetry also arises naturally in the "medicine dropper" problem. Again let $X$ denote the interior of the drop in contact with the horizontal hyperplane, $\Sigma$. Suppose that $\Sigma_{X}=$ $\bar{X} \cap \Sigma$ is a disk and the mean curvature of $\Omega$ is linear in height. The conjecture is that $X$ is contractable and axially symmetric. The following theorem covers this case.

THEOREM 1.2. Suppose $\Sigma$ is the hyperplane $\{u=0\}$ and let $X$ be a bounded, open, and connected subset of $R^{n+1}$ adhering to $\Sigma$. Suppose that $\Sigma_{X}=\Sigma \cap \bar{X}$ has nonempty interior in $\Sigma$ which is symmetic about an $(n-1)-p l a n e, \Pi$, in $\Sigma$ with the property that the boundary, $\Gamma$, of $\Sigma_{X}$ can be decomposed into two parts $\Gamma=\Gamma_{+} \cup \Gamma_{-}$ where $\Gamma_{+}$is the graph of a nonnegative $C^{2}$-function, $g$, from $\Pi_{X}=$ $\Sigma_{X} \cap \Pi$ such that $g$ is positive on the interior of its domain and vanishes on $\partial \Pi_{X} . \quad \Gamma_{-}$is the reflection of $\Gamma_{+}$about $\Pi$.

Suppose that the boundary of $X, \partial X=\Omega \cup \Sigma_{X}$ where $\Omega$ is a hy- 
persurface with boundary, $\Gamma=\partial \Omega=\Omega \cap \Sigma$, which is of class $C^{2}$ on its interior and on that part of $\partial \Omega$ not touching $\Pi$. Suppose also that the mean curvature, $H$, of $\Omega$ is the restriction to $\Omega$ of a $C^{1}$-function of $u$ alone (except perhaps on $\left.\Omega \cap \Pi\right)$. Let $T_{\Pi}$ be the vertical hyperplane generated by $\Pi$. Under these conditions $X$ is symmetric about $T_{\Pi}$ and the nonempty intersection of $X$ with any normal line to $T_{I I}$ is a line segment with center on $T_{I I}$.

Remark 1. Clearly, if $\partial \Omega \subset \Sigma$ is a circle we may apply Theorem 1.2 to conclude the axial symmetry of $X$ as asserted earlier.

Remark 2. If $\partial \Omega \subset \Sigma \equiv R^{2}$ is a square region with rounded corners we may conclude that $X$ is symmetric about the vertical hyperplanes generated by the two diagonals. However, if $\partial \Omega$ is a rectangle with smoothed corners, unequal sides, and $\ell_{1}, \ell_{2}$ are the lines of symmetry for $\partial \Omega$, then our theorem does not allow us conclude corresponding symmetry for $X$ about the generated vertical hyperplanes. (We do not consider the case when $\partial \Omega$ may have corners off of $I I$ although the arguments should work at least in certain cases.)

Remark 3. Consider the dumbell-shaped region $A_{\varepsilon} \subset \Sigma \equiv R^{2}$ consisting of the union of two disks $D_{i}=\left\{\left(x_{1}, x_{2}\right) \mid\left(x_{1}^{2} \pm 2\right)^{2}+x_{2}^{2} \leqq 1\right\}$ connected by a narrow neck $\left.R_{\varepsilon}=\left\{\left(x_{1}, x_{2}\right)\right\}-2 \leqq x_{1} \leqq 2,-\varepsilon \leqq x_{2} \leqq \varepsilon\right\}$, and suppose $X$ adheres to $\Sigma$ with $\Sigma_{X}=A_{\varepsilon}$ (again with rounded corners). The results of Theorem 1.2 assert that $X$ must be symmetric about the plane $x_{2}=0 . \quad A_{\varepsilon}$ is also symmetric about $x_{1}=0$. However, in this case $\partial A_{\varepsilon}$ cannot be represented by a graph plus its reflection and so we cannot conclude that $X$ is symmetric about $x_{1}=0$. In fact, for the case of no gravity, with $H=$ constant, and large volume for $X$, one would not expect the equilibrium configuration for $X$ of least area for $\Omega$ to possess such symmetry.

II. The touching principle. The theorems stated in this section are well-known results from the literature. We state them here for the sake of completeness and reference.

Let $w(x)=w\left(x_{1}, \cdots, x_{n}\right)$ be a differentiable function in some region of $R^{n}$. We shall write $w_{i} \equiv w_{i}\left(x_{1}, \cdots, x_{n}\right)$ to represent the partial derivative of $w$ with respect to $x_{i}$. Higher order derivatives are represented similarly.

Let $M(w)$ be a linear differential operator in some open set $G \subset R^{n}$.

$$
M(w)=\sum_{i, j} a_{i j}(x) w_{i j}+\sum_{i} b_{i}(x) w_{i}
$$


We assume that $a_{i j}(x)$ and $b_{i}(x)$ are continuous in $G$, that $a_{i j}(x)=$ $a_{j i}(x)$, with the summations in (2.1) for $1 \leqq i, j \leqq n . M(w)$ is elliptic on $G$ if

$$
\sum_{i, j} a_{i j}(x) \xi_{i} \xi_{j}>0 \text { for all } x \in G
$$

and $\xi=\left(\xi_{1}, \cdots, \xi_{n}\right) \neq(0, \cdots, 0)$. It is uniformly elliptic on $G$ with ellipticity constant $\kappa>0$ if

$$
\sum_{i, j} a_{i j}(x) \xi_{i} \xi_{j} \geqq \kappa|\xi|^{2} \quad \text { for all } x \in G .
$$

Lemma 2.1. (E. Hopf Maximum Principle [5]). Let $w \in C^{2}(G)$ satisfy $M(w) \geqq 0$ where $M$ is an elliptic operator on $G$. If there is a point $x_{0} \in G$ with $w\left(x_{0}\right) \geqq w(x)$ for all $x \in G$, then $w(x)$ is constant on $G$.

Lemma 2.2. (Hopf's Boundary Point Lemma [6]). Let $G$ be a region in $R^{n}$ and suppose that in a neighborhood of $x_{0} \in \partial G$, the boundary of $G$ is of class $C^{1}$. Let $M(w)$ be a uniformly elliptic operator on $\bar{G}$ and suppose that $w(x) \in C^{2}(G) \cap C^{1}(\bar{G})$ satisfies $M(w) \geqq 0$ on $G$. If $w\left(x_{0}\right) \geqq w(x)$ for all $x \in G$ then either $w(x)$ is a constant on $\bar{G}$ or the inward normal derivative $\partial w / \partial \nu<0$ at $x_{0}$.

Lemma 2.3. (Serrin's Boundary Point Lemma at a Corner [8]). Let $G \subset R^{n}$ be a bounded region which has a $C^{2}$ boundary in a neighborhood of $x_{0} \in \partial G$. Let $T$ be a normal plane to $\partial G$ at $x_{0}$ and let $G^{+}$be that component of $G$ lying on one side of $T$ which contains $x_{0}$ in its closure. Let $M(w)$ be a uniformly elliptic differential operator on $\bar{G}^{+}$which satisfies (2.3) for some $\kappa>0$ on $\bar{G}^{+}$. Suppose also that

$$
\left|\sum_{i, j} a_{i j}(x) \xi_{i} \eta_{j}\right| \leqq K[|(\xi \cdot \eta)|+|\xi| d]
$$

for some constant $K>0$, all $x \in \bar{G}^{+}$, any $\xi=\left(\xi_{1}, \cdots, \xi_{n}\right)$, where $\eta=$ $\left(\eta_{1}, \cdots, \eta_{n}\right)$ is a unit normal to $T$, and where $d$ is the distance from $x$ to $T$.

Let $w \in C^{2}\left(\bar{G}^{+}\right)$satisfy $M(w) \geqq 0$ on $\bar{G}^{+}$and suppose that $w\left(x_{0}\right) \geqq$ $w(x)$ for all $x \in \bar{G}^{+}$. If $w(x)$ is not constant on $\bar{G}^{+}$, then either $\partial w / \partial s<0$ or $\partial^{2} w / \partial s^{2}<0$ in any direction which enters $G^{+}$nontangentially at $x_{0}$.

There is a touching principle corresponding to each of these maximum principles. The proofs are well known and similar. We sketch the proof in the first instance. 
Lemma 2.4. (Interior Touching Principle). Let $M(w)$ be an elliptic operator on $G$ as described in Lemma 2.1. Suppose there is a function $w(x) \in C^{2}(G)$ which satisfies

$$
L(w) \equiv M(w)+c(x) w \geqq 0
$$

on $G$ where $c(x)$ is continuous. If $w(x) \leqq 0$ on $G$ and $w\left(x_{0}\right)=0$, then $w(x) \equiv 0$ on $G$.

Proof. It is sufficient to prove the lemma on any relatively compact neighborhood, $U$, of $x_{0}$ where $\bar{U} \subset G$. We set $w(x) \equiv e^{g x_{1}} u(x)$ where $\beta>0$ and $x_{1}$ is the first coordinate. A direct calculation yields $L(w)=e^{\beta x_{1}} \hat{L}(u)$ where $\hat{L}(u)=\widehat{M}(u)+\hat{c}(x) u$. Here $\hat{M}(u)$ has the form (2.1) with $\hat{a}_{i j}(x)=a_{i j}(x)$ and $\widehat{c}(x)=\beta^{2} a_{11}(x)+\beta a_{1}(x)+c(x)$. Thus $\hat{M}(u)$ is uniformly elliptic on $U$ and for large enough $\beta$, $\hat{c}(x)>0$ on $U$. Therefore $\hat{M}(u) \geqq-\widehat{c}(x) u \geqq 0$ on $U$, and $u(x)$ has a maximum at $x_{0}$ showing that $u(x)$ [and thus $w(x)$ ] is identically 0 on $U$.

Lemma 2.5. (Boundary Point Touching Principle). Let $G, x_{0}$ be as in Lemma 2.2. Suppose that $w(x) \in C^{2}(G) \cap C^{1}(\bar{G})$ satisfies (2.4) where $M(w)$ is a uniformly elliptic operator on $\bar{G}$ and $c(x)$ is continuous on $\bar{G}$. If $w\left(x_{0}\right)=0, w(x) \leqq 0$ for $x \in \bar{G}$, and the inward normal derivative $\partial w / \partial \nu=0$ at $x_{0}$, then $w$ is identically 0 .

Lemma 2.6. (Boundary Point Touching Principle at a Corner [8]). Let $G, G^{+}, T$, and $x_{0}$ be as in Lemma 2.3. Suppose that $w(x) \in C^{2}\left(\bar{G}^{+}\right)$satisfies the differential inequality (2.4) on $\bar{G}^{+}$where the uniformly elliptic operator, $M(w)$, on $\bar{G}^{+}$satisfies the conditions of Lemma 2.3 and $c(x)$ is continuous on $\bar{G}^{+}$. Let $w\left(x_{0}\right)=0, w(x) \leqq 0$ for $x \in G^{+}$, and suppose that for any nontangential direction entering $G^{+}$at $x_{0}$ we have $\partial w / \partial s=\partial^{2} w / \partial s^{2}=0$. Then $w(x)$ vanishes on $\bar{G}^{+}$.

Now let $u(x)$ and $v(x)$ be two solutions to the same prescribed mean curvature equation

$$
\operatorname{div}(T u)=n H(x, u), \quad T u=\nabla u /\left(1+|\nabla u|^{2}\right)
$$

in a region, $G$. The operator $\operatorname{div}(T u)$ is quasi-linear and may be written in the form

$$
\begin{aligned}
\operatorname{div}(T u) & =\sum_{i, j} a_{i j}(x, u, \nabla u) u_{i j} \\
& =(1 / W) \sum_{i} u_{i i}-\left(1 / W^{3}\right) \sum_{i, j} u_{i} u_{j} u_{i j}
\end{aligned}
$$

where $W^{2}=1+|\nabla u|^{2} \equiv 1+|p|^{2}$. It follows that 
(2.7)

$$
\sum_{i, j} a_{i j}(x, u, \nabla u) \xi_{i} \xi_{j}=\left(1 / W^{3}\right)\left[\left(1+p^{2}\right)|\xi|^{2}-\sum_{i, j} p_{i} p_{j} \xi_{i} \xi_{j}\right] \geqq\left(1 / W^{3}\right)|\xi|^{2} .
$$

Now let $w(x)=u(x)-v(x)$. Then, as is well known, $w(x)$ is a solution to a homogeneous linear elliptic P.D.E. of the form $M(w)+$ $c(x) w \equiv 0$. Here $M(w)$ is in the form (2.1) and the principle part of $M(w)$ is

$$
a_{i j}(x)=\int_{0}^{1} a_{i j}(x, u+t(v-u), \nabla u+t(\nabla v-\nabla u)) d t
$$

where $a_{i j}(x, u, p)$ is given by (2.6) and (2.7). In particular, $M(w)$ is elliptic and on any bounded domain is uniformly elliptic with ellipticity constant

$$
\kappa=1 / \max \left(W_{0}^{3}, W_{1}^{3}\right)
$$

where $W_{0}^{2}(x)=1+|\nabla u|^{2}$ and $W_{1}^{2}(x)=1+|\nabla v|^{2}$.

These remarks lead to the following conclusions.

Application 1. Let $u(x)$ and $v(x)$ be two $C^{2}$ solutions to the same differential equation of prescribed mean-curvature, (2.5), on a region $G \subset R^{n}$ where $H(x, u)$ is continuously differentiable on $G \times R$. Suppose that $u(x) \leqq v(x)$ on $G$ and $u\left(x_{0}\right)=v\left(x_{0}\right)$ for some $x_{0} \in G$. Then $u(x) \equiv v(x)$ on $G$.

Application 2. Let $G, x_{0}$ be as in Lemma 2.2. Suppose that $u(x)$ and $v(x) \in C^{1}(\bar{G}) \cap C^{2}(G)$ are both solutions to the same prescribed mean-curvature differential equation, (2.5), where $H(x, u)$ is continuously differentiable on $\bar{G} \times R$. If $u\left(x_{0}\right)=v\left(x_{0}\right), u(x) \leqq v(x)$ for $x \in G$, and the inward normal derivatives $\partial u / \partial \nu=\partial v / \partial \nu$ at $x_{0}$, then $u(x) \equiv v(x)$ on $\bar{G}$.

Application 3. Let $G, G^{+}, T$ and $x_{0}$ be as in Lemma 2.3. Suppose that $u(x)$ and $v(x) \in C^{2}\left(\bar{G}^{+}\right)$are both solutions to the same prescribed mean-curvature differential equation, (2.5), on $\bar{G}^{+}$where $H(x, u)$ is continuously differentiable on $\bar{G}^{+} \times R$. If $u\left(x_{0}\right)=v\left(x_{0}\right)$, $u(x) \leqq v(x)$ for $x \in \bar{G}^{+}$, and if for any nontangential direction pointing into $G^{+}$at the corner $x_{0}$ we have $\partial u / \partial s=\partial v / \partial s$ and $\partial^{2} u / \partial s^{2}=$ $\partial^{2} v / \partial s^{2}$, then $u(x) \equiv v(x)$ on $\bar{G}^{+}$.

\section{Proofs of the main theorems.}

Proof of Theorem 1.1. Following the procedure of Alexandrov and Serrin we let $T_{0}$ be a vertical hyperplane in $R^{n+1}$ which lies outside of $\bar{X}$. We move $T_{0}$ through a one-parameter family of parallel hyperplanes, $T$, towards and into $X$. Once $T$ has cut into 
$X$, let $\Omega^{\prime}(T)$ be the reflection about $T$ of that part of $\Omega$ through which $T$ has passed. We adopt the convention that $\Omega^{\prime}(T)$ is closed so that $\Omega \cap T \subset \Omega^{\prime}(T)$. Similarly, we let $\Omega(T)$ be that part of $\Omega$ through which $T$ has not passed. Again $\Omega(T)$ is assumed closed so that $\Omega \cap T \subset \Omega(T)$.

When $T$ first cuts into $X$, the interior of $\Omega^{\prime}(T)$ will be contained in $X$. For $0 \leqq \alpha \leqq \pi$, this will continue to be true until at least one of the following possibilities occur for some $T=T_{1}$.

1. $\Omega^{\prime}\left(T_{1}\right)$ will be internally tangent to $\Omega\left(T_{1}\right)$ at a point, $P$, off of $\Sigma$ and away from $T_{1}$.

2. At some point, $P$, on $\Omega \cap T_{1}$ but off of $\Sigma$ the normal, $n(P)$, to $\Omega$ at $P$ will be parallel to $T_{1}$.

3. $\Omega^{\prime}\left(T_{1}\right)$ will touch $\Omega\left(T_{1}\right)$ internally at a point, $P$, on $\Sigma$ but away from $T_{1}$.

4. At some point, $P$, on $\Omega \cap T_{1}$ lying on $\Sigma$ the exterior normal, $m(P)$, to $\partial \Omega \equiv \Gamma$ in $\Sigma$ will be parallel to $T_{1}$.

We first show that there is a first time, $T=T_{1}$, where at least one of these possibilities occur.

For each $Q \in T_{0}$ let $\iota(Q)$ be the normal half line to $T_{0}$ from $Q$ directed towards $X$. Let $P_{1}$ be the initial contact point of $\ell(Q)$ with $\Omega$ if such exists. Now set $a(Q)$ to be the distance from $Q$ to $P_{1}$ if $P_{1}$ exists, otherwise set $a(Q)=+\infty . a(Q)$ is a lower semi-continuous function on $T_{0}$.

Next let $Q \in T_{0}$ be a point off of $\Sigma$ such that $\ell(Q)$ meets $\Omega$. If $\ell(Q)$ cuts through $\Omega$ transversally at $P_{1}$, let $P_{2}$ be the second time that $\angle(Q)$ meets $\Omega$ and set $b(Q)$ to be the distance from $Q$ to $P_{2}$. If the normal, $n\left(P_{1}\right)$, to $\Omega$ at $P_{1}$ is parallel to $T_{0}$ then set $P_{2}=P_{1}$ and $b(Q)=a(Q)$. Again, if $\ell(Q)$ fails to meet $\Omega$, set $b(Q)=+\infty$.

Now suppose $Q \in T_{0} \cap \Sigma$ with $\ell(Q)$ meeting $\Omega$ for the first time at $P_{1}$. Suppose that the normal, $m\left(P_{1}\right)$, to $\partial \Omega$ in $\Sigma$ is not parallel to $T_{0}$. If $0<\alpha<\pi$ then the normal vector, $n\left(P_{1}\right)$, to $\Omega$ at $P_{1}$ also is not parallel to $T_{0}$. It follows that $\ell(Q)$ will cut through $\Omega$ and there will be a second point, $P_{2}$, where $\ell(Q)$ meets $\Omega$. Observe that this will remain true for points $Q^{\prime} \in T_{0}$ near $Q$ for which $\ell(Q)$ meets $\Omega$. As above we set $b(Q)$ to be the distance from $Q$ to $P_{2}$.

Now suppose that $\alpha=0$ or $\alpha=\pi$. In this case we observe that the prescribed mean-curvature function, $H(u)$, for $\Omega$ must satisfy $H(0) \neq 0$. If $H(0)=0$, it would follow that in a neighbornood of $P_{1}$ both $\Omega$ and $\Sigma$ could be expressed nonparametrically in the form $u=u(x)$ as solutions to the same prescribed mean-curvature equation (2.5). It follows from Application 2 that $\Omega \equiv \Sigma$, a contradiction. Since $\alpha=0$ or $\pi$ and the mean curvature of $\Omega$ at $P_{1}$ is not zero it follows that the normal curvature, $k\left(P_{1}\right)$ of $\Omega \cap N$ where $N$ is the normal 2-plane to $\partial \Omega$ at $P_{1}$, is different from zero. 
This implies that for points $P^{\prime}$ near $P_{1}$ on $\Omega$ but off of $\Sigma$, the normal vector, $n\left(P^{\prime}\right)$, to $\Omega$ at $P^{\prime}$ is not parallel to $T_{0}$. Once again this means that for all points $Q^{\prime}$ near $Q$ on $T_{0}$ any directed normal line, $\ell\left(Q^{\prime}\right)$, which meets $\Omega$ will cut through $\Omega$ and thus will meet a second time at a point, $P_{2}$. As above we let $b(Q)$ to be the distance from $Q$ to $P_{2}$. Finally, if $m\left(P_{1}\right)$ is parallel to $T_{0}$ then set $P_{2}=P_{1}$ and $a(Q)=b(Q)$.

Our discussion allows us to conclude that $b(Q)$ is a lower semicontinuous function on $T_{0}$. Now let $c(Q)=[a(Q)+b(Q)] / 2 . c(Q)$ is also lower semi-continuous and so there is a point $Q^{*} \in T_{0}$ where $c(Q)$ takes on a positive minimum. This minimum value is precisely the distance through which we must move $T_{0}$ to reach the hyperplane, $T_{1}$, where at least one of the conditions (1)-(4) first apply.

We now consider each of the four possibilities.

Possibility 1. Choose a Euclidean coordinate system $\left(x_{1}, \cdots\right.$, $\left.x_{n}, u\right)$ with the origin at $P$ such that the tangent space to $\Omega$ at $P$ is $u=0$ and so that the $u$-axis is directed into $X$. In a neighborhood of $x=\bar{O}$ both $\Omega\left(T_{1}\right)$ and $\Omega^{\prime}\left(T_{1}\right)$ may be represented nonparametrically in the form $u(x)$ and $v(x)$ respectively where both functions satisfy the same prescribed mean-curvature equation, (2.5), for some $C^{\prime}$-function $H(x, u)$. We also have $u(\bar{O})=v(\bar{O}), u(x) \leqq v(x)$ and so by Application $1 u(x) \equiv v(x)$ and $\Omega\left(T_{1}\right)=\Omega^{\prime}\left(T_{1}\right)$.

Possibility 2. Choose a Euclidean coordinate system to that $P$ is the origin, $u=0$ is the tangent space to $\Omega$ at $P$, the hyperplane, $T_{1}$, is given by $x_{1}=0$, with the positive $u$-axis pointing into $X$, and the positive $x_{1}$-axis pointing towards $\Omega^{\prime}\left(T_{1}\right)$. There is a neighborhood, $U$, of the origin in $\left(x_{1}, \cdots, x_{n}\right)$ space such that on the domain $G=\bar{U} \cap\left\{x_{1} \geqq 0\right\}$ both $\Omega\left(T_{1}\right)$ and $\Omega^{\prime}\left(T_{1}\right)$ may be represented nonparametrically by $C^{2}$-functions $u(x)$ and $v(x)$ both satisfying the same prescribed mean-curvature equation, (2.5), on $\bar{G}$. By construction we have $u(\bar{O})=v(\bar{O}), \quad u(x) \leqq v(x)$ for $x \in \bar{G}$, and $\partial u / \partial x_{1}=$ $\partial v / \partial x_{1}=0$ at $x=\bar{O}$. By Application 2 it follows that $u(x) \equiv v(x)$ on $\bar{G}$ and so $\Omega\left(T_{1}\right)=\Omega^{\prime}\left(T_{1}\right)$.

Possibility 3. The argument is similar to Possibility 2. Choose a Euclidean coordinate system $\left(x_{1}, \cdots, x_{n}, u\right)$ centered at $P$ so that $u=0$ is the tangent space to $\Omega$ at $P$, with $u=x_{1}=0$ the tangent space to $\partial \Omega$ at $P$ lying in $\Sigma$, so that the positive $x_{1}$-axis is directed towards $\Omega$, and the positive $u$-axis heads into $X$.

Since the angle of contact, $\alpha$, is constant along $\partial \Omega$ it follows that the hyperplane $u=0$ is the common tangent space to $\Omega\left(T_{1}\right)$ and $\Omega^{\prime}\left(T_{1}\right)$ at $P$. Since $\Omega$ is of class $C^{2}$ with boundary, it follows 
that in a neighborhood of $P, \Omega\left(T_{1}\right)$ and $\Omega^{\prime}\left(T_{1}\right)$ are represented nonparametrically by $C^{2}$-functions $u(x)$ and $v(x)$ respectively where $u(x)$ and $v(x)$ are defined on domains $G_{1}, G_{2} \subset R^{n}$ where $\partial G_{i}$ is a $C^{2}$-surface in $R^{n}$ containing the origin with the $x_{1}$-axis normal to $\partial G_{i}$ at $x=\bar{O}$ and pointing into $G_{i}$. From our construction we have $G_{1} \supset G_{2}$ if $O \leqq \alpha<\pi / 2, G_{1}=G_{2}=\left\{x_{1}>0\right\}$ if $\alpha=\pi / 2$, and $G_{1} \subset G_{2}$ if $\alpha>\pi / 2$. We let $G=G_{1} \cap G_{2}$ and use Application 2 again. On $\bar{G}, u(x)$ and $v(x)$ are solutions to the same prescribed mean-curvature equation, (2.5), with $u(\bar{O})=v(\bar{O}), u(x) \leqq v(x)$ for $x \in \bar{G}$, and $\partial u / \partial x_{1}=\partial v / \partial x_{1}=0$ at $x=\bar{O}$. By Application 2 we conclude that $u(x) \equiv v(x)$ and hence $\Omega\left(T_{1}\right)=\Omega^{\prime}\left(T_{1}\right)$.

Possibility 4. Choose a Euclidean coordinate system $\left(x_{1}, \cdots, x_{n}, x\right)$ with the origin at $P$, so that $u=0$ is the tangent space to $\Omega$ at $P$ with the positive $u$-axis directed into $X$, so that $x_{1}=0$ is the reflecting plane, $T_{1}$, with the positive $x_{1}$-axis pointing towards $\Omega\left(T_{1}\right)$, and so that $x_{n}=u=0$ is the tangent space to $\partial \Omega$ at $P$ in $\Sigma$ with the positive $x_{n}$-axis directed towards $\Omega$.

Relative to this coordinate system the surface, $\Omega$, in a neighborhood of $P$, is represented nonparametrically by a function $u(x)$ of class $C^{2}$ on a domain $\bar{G} \subset R^{n}$ where $\bar{O} \in \partial G$ and $\partial G$ is the graph of a $C^{2}$-function, $x_{n}=\phi\left(x_{1}, \cdots, x_{n-1}\right)$ satisfying $\dot{\phi}(\bar{O})=0, \dot{\phi}_{j}(\bar{O})=0$ for $j=1, \cdots, n-1$, and $G$ lies above the graph of $\phi(x) . \Omega\left(T_{1}\right)$ is represented by this function, $u(x)$, on $\bar{G}_{1}^{+} \equiv \bar{G} \cap\left\{x_{1}>0\right\}$ while the reflected surface, $\Omega^{\prime}\left(T_{1}\right)$, is represented by the function $v(x)$ on $\bar{G}_{2}$. where $v\left(x_{1}, x_{2} \cdots, x_{n}\right)=u\left(-x_{1}, x_{2}, \cdots, x_{n}\right)$ for $x_{1} \geqq 0$ and $\bar{G}_{2}^{+}$is the reflection of $\bar{G}^{-}$about $x_{1}=0$. Observe that $\bar{G}_{2}^{+} \subset \bar{G}_{1}^{+}$if $0 \leqq \alpha<\pi / 2$, $\bar{G}_{1}^{+}=\bar{G}_{2}^{+}$if $\alpha=\pi / 2$, and $\bar{G}_{1}^{+} \subset \bar{G}_{2}^{+}$if $\pi / 2<\alpha \leqq \pi$.

If we let $\bar{G}^{+}=\bar{G}_{1}^{+} \cap \bar{G}_{2}^{+}$, then $u(x)$ and $v(x)$ are both $C^{2}\left(\bar{G}^{+}\right)$solutions to the same precribed mean-curvature equation (2.5). Furthermore, $u(\bar{O})=v(\bar{O}), u(x) \leqq v(x)$ for $x \in \bar{G}^{+}$, and $\partial u / \partial s=\partial v / \partial s=0$ at $x_{0}=\bar{O}$ in any nontangential direction entering $G^{+}$.

We now show that $\partial^{2} u / \partial s^{2}=\partial^{2} v / \partial s^{2}$ at $x_{0}=\bar{O}$ in any nontangential direction entering $G^{+}$. It suffices to show that $u_{i j}(\bar{O})=v_{i j}(\bar{O})$ for $1 \leqq i, j \leqq n$. From the definition of $v(x)$ it follows at once that $u_{2 j}(\bar{O})=v_{i j}(\bar{O})$ for $2 \leqq i, j \leqq n$ or if $i=j=1$. Since $u\left(x_{1}, x_{2}, \cdots\right.$, $\left.x_{n}\right) \leqq u\left(-x_{1}, x_{2}, \cdots, x_{n}\right)$ when $x_{1} \geqq 0$, it follows that $u_{1}\left(0, x_{2}, \cdots, x_{n}\right) \leqq 0$ and so $u_{1 j}(\bar{O})=v_{1 j}(\bar{O})=0$ for $j=2, \cdots, n-1$. Since $x_{n} \geqq 0$ for $x \in G$ we must argue differently for $u_{1 n}(\bar{O})$.

However, $\Omega$ intersects the hyperplane, $\Sigma$, at a constant angle, $\alpha$. The unit normal to $\Omega$ is given by $N=(\nabla u,-1) / W$ and the unit normal to $\Sigma$ may be written $\xi=(0, \cdots, 0, b, a)$ where $a^{2}+b^{2}=1$. It follows that $\cos \alpha=\left(b u_{n}-a\right) / W$. Substitute $x_{n}=\dot{\rho}\left(x_{1}, \cdots, x_{n-1}\right)$ 
into this equation and differentiate with respect to $x_{1}$. We find $0=b\left[u_{1 n}(\bar{O})+u_{n n}(\bar{O}) \phi_{1}(\bar{O})\right]$. Since $\phi_{1}(\bar{O})=0$ we conclude that $u_{1 n}(\bar{O})=0$ if $b \neq 0$. However, if $b=0$ then $\alpha=0$ or $\alpha=\pi$. In this case we have $u_{n}\left(x_{1}, \cdots, x_{n-1}, \phi\left(x_{1}, \cdots, x_{n-1}\right)\right) \equiv 0$. Again differentiate this expression with respect to $x_{1}$, set $x=\bar{O}$ and we find that $u_{1 n}(\bar{O})=$ $v_{1 n}(\bar{O})=0$ in this case also.

We have verified all the conditions of Application 3. We conclude that $u(x)=v(x)$ for $x \in G$ and hence $\Omega\left(T_{1}\right)=\Omega^{\prime}\left(T_{1}\right)$.

Proof of Theorem 1.2. Let $T_{0} \subset R^{n+1}$ be a vertical hyperplane which is exterior $\bar{X}$ and parallel to $T$. As in the proof of Theorem 1.1 we consider the possibility of moving $T_{0}$ through the one-parameter of hyperplanes, $T$, parallel to $T_{0}$ into $X$. For $Q \in T_{0}$ we define the functions $a(Q)$ and $b(Q)$ as previously if $Q$ is off of $\Sigma$. Let $Q \in T_{0} \cap \Sigma$ and suppose the normal half line, $\ell(Q)$, intersects $\Omega$. If $\ell(Q)$ first meets $\partial \Omega$ at a point, $P_{1}$, off of $\Pi$ then, since $\partial \Omega$ is represented by a graph at $P_{1}$, the normal, $m\left(P_{1}\right)$, to $\partial \Omega$ in $\Sigma$ is not parallel to $T_{0}$ and so $\ell(Q)$ will meet $\Omega$ a second time at a point, $P_{2}$. We set $a(Q)=d\left(Q, P_{1}\right)$ and $b(Q)=d\left(Q, P_{2}\right)$. If $\ell(Q)$ first meets $\Omega$ at a point $P_{1}$ on $I I$ then we set $b(Q)=a(Q)=d\left(Q, P_{1}\right)$. As before, it follows that both $a(Q)$ and $b(Q)$ are lower semi-continuous functions which implies that $[a(Q)+b(Q)] / 2$ takes on a positive minimum.

Let $T_{1}$ be the corresponding hyperplane. If $T_{1}$ is not $T_{I I}$ then it follows that either Possibility 1 or 2 occurs at a point $P \in \Omega$ and $P \notin \Sigma$. By the appropriate touching principle, Application 1 or 2, we conclude that $\Omega\left(T_{1}\right)=\Omega^{\prime}\left(T_{1}\right)$, an impossibility unless $T_{1}=T_{l l}$. Therefore $T_{1}=T_{\Pi}$.

The same conclusion must hold if we had initially chosen $T_{0}$ to lie on the other side of $T_{\Pi}$. The only way for this to be true is if $X$ itself is symmetric about $T_{I}$ and such that any nonempty intersection of $X$ with a normal line through $T_{I T}$ is a segment whose center lies on $T_{\Pi I}$.

\section{REFERENCES}

1. A. D. Alexandrov, Uniqueness theorems for surfaces in the large, V. Vestnik Leningrad University 13, No. 19 (1958), 5-8. Am. Math. Soc. Translations (Series 2) 21, 412-416.

2. P. Concus and R. Finn, The shape of a pendent liquid drop, Philos. Trans. Roy. Soc. London Ser. A, 292 (1979), 307-340.

3. R. Finn, The liquid sessile drop, Pacific J. Math., (this issue).

4. E. Gonzalez, Sul problema della goccia appoggiata, Rend. Sem. Mat. Univ. Padova, 55 (1976), 289-302.

5. E. Hopf, Elementare Bemerkungen über die Lösungen partielle differential-gleichungen zwiter ordnung vom elliptischen typus, Sita.-ber. Preuss. Akad. Wiss., 19 (1927), 147-152. 6 . - A remark on linear elliptic differential equations of the second order, Proc. Amer. Math. Soc., 3 (1952), 791-793. 
7. J. Serrin, On surfaces of constant mean curvature which span a given space curve, Math. Z., 112 (1969), 77-88.

8. - A symmetry problem in potential theory, Arch. Rat. Mech. and Anal., 43 (1971), 304-318.

9. H. C. Wente, The stability of the axially symmetric pendent drop, Pacific J. Math., (this issue).

Received November 1, 1979. A substantial portion of this paper was completed while the author was a Visiting Member of SFB 40 at the University of Bonn, Germany.

UNIVERSITY OF TOLEDO

Toledo, OH 43606 



\section{PACIFIC JOURNAL OF MATHEMATICS}

\section{EDITORS}

DONALD BABBITT (Managing Editor)

University of California

Los Angeles, CA 90024

Hugo Rossi

University of Utah

Salt Lake City, UT 84112

C. C. MOORE and ANDREW OGG

University of California

Berkeley, CA 94720
J. DUGUNDJI

Department of Mathematics

University of Southern California

Los Angeles, CA 90007

R. FinN and J. Milgram

Stanford University

Stanford, CA 94305

\section{ASSOCIATE EDITORS}
E. F. BECKENBACH
B. H. NeumanN
F. WOLF
K. YoSHIDA

\section{SUPPORTING INSTITUTIONS}

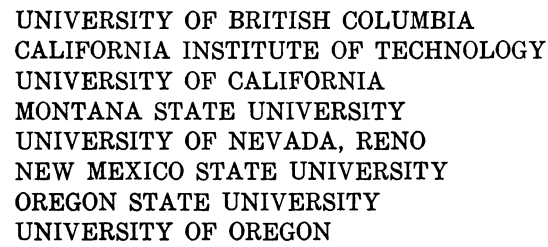

UNIVERSITY OF BRITISH COLUMBIA CALIFORNIA INSTITUTE OF TECHNOLOGY UNIVERSITY OF CALIFORNIA MONTANA STATE UNIVERSITY UNIVERSITY OF NEVADA, RENO NEW MEXICO STATE UNIVERSITY OREGON STATE UNIVERSITY UNIVERSITY OF OREGON

\author{
UNIVERSITY OF SOUTHERN CALIFORNIA \\ STANFORD UNIVERSITY \\ UNIVERSITY OF HAWAII \\ UNIVERSITY OF TOKYO \\ UNIVERSITY OF UTAH \\ WASHINGTON STATE UNIVERSITY \\ UNIVERSITY OF WASHINGTON
}

The Supporting Institutions listed above contribute to the cost of publication of this Journal, but they are not owners or publishers and have no responsibility for its content or policies.

Mathematical papers intended for publication in the Pacific Journal of Mathematics should be in typed form or offset-reproduced, (not dittoed), double spaced with large margins. Please do not use built up fractions in the text of the manuscript. However, you may use them in the displayed equations. Underline Greek letters in red, German in green, and script in blue. The first paragraph or two must be capable of being used separately as a synopsis of the entire paper. Please propose a heading for the odd numbered pages of less than 35 characters. Manuscripts, in triplicate, may be sent to any one of the editors. Please classify according to the scheme of Math. Reviews, Index to Vol. 39. Supply name and address of author to whom proofs should be sent. All other communications should be addressed to the managing editor, or Elaine Barth, University of California, Los Angeles, California, 90024.

50 reprints to each author are provided free for each article, only if page charges have been substantially paid. Additional copies may be obtained at cost in multiples of 50 .

The Pacific Journal of Mathematics is issued monthly as of January 1966. Regular subscription rate: $\$ 84.00$ a year (6 Vols., 12 issues). Special rato: $\$ 42.00$ a year to individual members of supporting institutions.

Subscriptions, orders for numbers issued in the last three calendar years, and changes of address shoud be sent to Pacific Journal of Mathematics, P.O. Box 969, Carmel Valley, CA 93924, U.S.A Old back numbers obtainable from Kraus Periodicals Co., Route 100, Millwood, NY 10546.

PUBLISHED BY PACIFIC JOURNAL OF MATHEMATICS, A NON-PROFIT CORPORATION

Printed at Kokusai Bunken Insatsusha (International Academic Printing Co., Ltd.). 8-8, 3-chome, Takadanobaba, Shinjuku-ku, Tokyo 160, Japan. 


\section{Pacific Journal of Mathematics \\ Vol. 88, No. $2 \quad$ April, 1980}

Reinhold Böhme, Stefan Hildebrandt and Engelbert Tausch, The two-dimensional analogue of the catenary ................. 247

Jean Ellen Taylor, Nonexistence of F-minimizing embedded disks ........ 279

Claus Gerhardt, A free boundary value problem for capillary surfaces ..... 285

Enrico Giusti, Generalized solutions for the mean curvature equation . . . . . 297

Jin-Tzu Chen, On the existence of capillary free surfaces in the absence of gravity.................................... 323

Leon M. Simon, Regularity of capillary surfaces over domains with corners.......................................... 363

Nicholas Jacob Korevaar, On the behavior of a capillary surface at a re-entrant corner.................................... 379

Henry Wente, The symmetry of sessile and pendent drops ............ 387

E. Gonzalez, Umberto Massari and I. Tamanini, Existence and regularity for the problem of a pendent liquid drop ...................... 399

Henry Wente, The stability of the axially symmetric pendent drop ........ 421

David Siegel, Height estimates for capillary surfaces . . . . . . . . . . . . 471

Bruce Edward Turkington, Height estimates for exterior problems of capillarity type ................................ 517

Robert Finn, The sessile liquid drop. I. Symmetric case .............. 541 